\title{
VARIACIÓN DE LA BETA-2 MICROGLOBULINA EN ORINA FETAL EN PUNCIONES SUCESIVAS PREVIO A DERIVACIÓN VESICOAMNIÓTICA ANTENATAL
}

\author{
Enrique Gil Guevara1 ${ }^{1}$ Ramiro Diaz ${ }^{1,2}$, Carlos Bermudez².
}

\begin{abstract}
RESUMEN
Antecedentes y objetivos. La uropatía obstructiva fetal puede ser tratada precozmente mediante la colocación de derivación vesicoamniótica intrauterina. Los criterios de tratamiento incluyen la ausencia de malformaciones mayores asociadas, cariotipo normal y función renal conservada demostrada por beta-2 microglobulina $<4 \mathrm{mg} / \mathrm{dl}$. Por la capacidad de concentración de orina en la vejiga fetal, si en una primera punción el valor de beta 2 microglobulina es superior al punto de corte se requiere una segunda punción a las 48-72 horas para verificar el valor en orina nueva, no concentrada que correspondería al valor real. El objetivo de investigación es determinar el comportamiento de la variación de los niveles de beta 2 microglobulina, en 2 muestras seriadas de orina fetal con intervalo de 48 - 72 horas, en fetos con diagnóstico de uropatía obstructiva referidao al Centro Médico Docente La Trinidad, para establecer la posibilidad de omisión de la segunda punción en caso de encontrarse un patrón específico de comportamiento de la disminución de los valores. Métodos. Se realizó un estudio clínico unicéntrico, descriptivo y longitudinal, incluyendo a 15 embarazadas cuyos fetos fueron diagnosticados con uropatía obstructiva y sin cromosopatías, practicándoseles vesicocentesis seriada (48 a 72 horas) para biometría urinaria. Resultados. Los valores de beta 2 microglobulina resultaron $>4 \mathrm{mg} / \mathrm{dl}$ en los 15 casos con la primera vesicocentesis, y disminuyeron a $<4 \mathrm{mg} / \mathrm{dl}$ en 7 casos $(46,6 \%$ ) en la segunda vesicocentesis. En todos los casos cuando la beta 2 microglobulina fue $<8 \mathrm{mg} / \mathrm{dl}$ en la primera vesicopunción, siempre disminuyó a valores muy próximos a $4 \mathrm{mg} / \mathrm{dl}$ (máximo de 4,3 mg/dl) en la segunda vesicopunción. Conclusiones. Se plantea la colocación in utero de derivaciones vesicoamnióticas en los casos de uropatía obstructiva fetal cuando la beta 2 microglobulina sea $<8 \mathrm{mg} / \mathrm{dl}$ en la primera vesicopunción, omitiendo realizar una segunda vesicopunción.
\end{abstract}

Palabras clave: Uropatía obstructiva fetal; Beta 2 microglobulina; Terapia de derivación vesicoamniotica (Fuente DeCS BIREME).

\section{PROGNOSTIC VALUE OF FETAL URINARY BETA-2 MICROGLOBULINE IN VESICOAMNIOTIC SHUNTING THERAPY FOR FETAL OBSTRUCTIVE UROPATHY}

\begin{abstract}
Introduction and objectives. Fetal obstructive uropathy can be treated early in pregnancy by intrauterine vesicoamniotic shunting, immediately after being diagnosed by ultrasound, in specific cases with a favorable prognosis in renal function using fetal urinary beta-2 microglobuline. The main objective of the study was to determine the changes of beta-2 microglobuline in consecutive samples of fetal urine in $48-72$ hour-intervals in fetuses with obstructive uropathy at 16 weeks of gestation. Methods. We performed a descriptive and longitudinal study, including 15 pregnant women whose fetuses were diagnosed with obstructive uropathy and without chromosomal abnormalities, performing vesicocentesis for urinary biometry. Results. Beta-2 microglobuline values were $>4 \mathrm{mg} / \mathrm{dl}$ in the first vesicocentesis of all 15 cases and decreased to $<4 \mathrm{mg} / \mathrm{dl}$ in 7 cases $(46.6 \%)$ after second vesicocentesis. In all cases when beta-2 microglobuline was $<8 \mathrm{mg} / \mathrm{dl}$ in the first vesicocentesis, there was a decrease to nearly $4 \mathrm{mg} / \mathrm{dl}$ (maximum $4.3 \mathrm{mg} / \mathrm{dl}$ ) in the second vesicocentesis. Conclusions. Vesicoamniotic shunting should be performed in all cases of fetal obstructive uropathy when the values of beta- 2 microglobuline were $<8 \mathrm{mg} / \mathrm{dl}$ at the first vesicocentesis.
\end{abstract}

Key words: Fetal obstructive uropathy; Beta 2 microglobuline; Vesicoamniotic shunt therapy (Source: MeSH NLM).

\section{INTRODUCCIÓN}

La obstrucción de las vías urinarias inferiores ocurre aproximadamente en 1:6.000 nacidos vivos. Sin tratamiento esta alteración suele conducir a hidronefrosis bilateral, displasia renal, oligohidramnios o anhidramnios, hipoplasia pulmonar, deformidades fetales por compresión extrínseca y muerte perinatal ${ }^{1}$.

Las causas de obstrucción urinaria baja generalmente corresponden a síndrome de valvas uretrales posteriores, atresia uretral y síndrome de Prune Belly. El ultrasonido es la base del diagnóstico prenatal ${ }^{2}$. El hallazgo ecográfico característico en casos de síndrome de valvas posteriores es la dilatación del tercio proximal de la uretra y megavejiga; concomitantemente puede evidenciarse hidrouréteres, dilatación pielocalicial e hidronefrosis bilateral. Además, el oligo o anhidramnios resultante del proceso obstructivo puede originar la secuencia de Potter, un síndrome incompatible con la vida, cuyos hallazgos se asocian a insuficiencia renal en el feto y a la ausencia de líquido amniótico, cursando con severas malformaciones

Center for Fetal Cellular \& Molecular Therapy, Cincinnati Fetal Center, Cincinnati Children's Hospital Medical Center, Cincinnati, OH, United States.

Centro Medico Docente La Trinidad, Caracas Venezuela. 
urinarias, hipoplasia pulmonar, anomalías craneofaciales y de las extremidades ${ }^{3}$.

Las técnicas de manejo terapéutico para la uropatía obstructiva fetal son la colocación intraútero de derivaciones o shunts vesicoamnióticos en el feto, técnica que se propuso en 1982, la cual actúa como una medida descompresiva para la vía urinaria. Desde entonces se han definido con mayor precisión las pautas para la selección específica de aquellos casos a los que se les realizará el procedimiento quirúrgico in útero con fines terapéuticos ${ }^{4}$

Para poder incorporarse a alguno de los diversos protocolos de manejo terapéutico, es básico realizar un estudio genético fetal y tener función renal conservada. El mejor parámetro pronóstico de la función renal es la demostración de beta 2 microglobulina en orina fetal inferior a $4 \mathrm{mg} / \mathrm{dl}$, lo cual se asocia a cerca de $100 \mathrm{de}$ funcionalismo renal conservado. La orina fetal se puede concentrar en la vejiga urinario, por lo cual el hecho de obtenerse valores de la proteína superiores al punto de corte pudiera corresponder a un falso negativo, por lo cual si el valor excede al punto de corte se requiere una segunda punción (48 a 72 horas), para asegurarse del valor real de beta 2 microglobulina. Si en una segunda vesicocentesis el valor es inferior al punto de corte el feto plica para tratamiento, si por el contrario el valor de la proteína es superior al punto de corte entonces el feto no se beneficiará del tratamiento, ya que se presentará falla renal, anhidramnios e hipoplasia pulmonar ${ }^{4}$.

Asimismo, la función renal fetal puede ser evaluada a través de ultrasonido; sin embargo, el resultado logrado utilizando ese método diagnóstico para dicho objetivo es muy poco específico, por lo que se recomienda preferiblemente la vesicocentesis para obtener muestras de orina fetal y realizar el análisis correspondiente ${ }^{4}$.

\section{Problema a estudiar}

Considerando el diagnóstico de uropatía obstructiva fetal, la atención prenatal apropiada depende de la capacidad para determinar con seguridad la presencia o extensión de daño funcional en los riñones, de tal manera que puedan ser seleccionados los casos que pudieran beneficiarse del tratamiento quirúrgico para prevenir el daño funcional renal.

Uno de los procedimientos diagnósticos disponible es el análisis de la orina fetal, teniendo en cuenta que la obtención de niveles de beta 2 microglobulina en primera punción superiores al punto de corte, no significa necesariamente que se trate de un caso de deterioro de la función renal, ya que la elevación puede ser consecuencia de aumento de la concentración de la orina que ocurre en la vejiga, por lo cual será requerida una segunda punción para obtener orina nueva. con intervalo de $48-72$ horas para la determinación de beta 2 microglobulina, lo que permitirá establecer un tipo claro de disminución de hipertonicidad; sin embargo, este método presenta riesgos que pueden afectar a la madre y al feto.

En el presente estudio se determina el patrón de comportamiento de la disminución de los niveles de beta 2 microglobulina mediante el análisis seriado de la orina fetal, verificando la modificación de la misma entre la primera y segunda toma, con la finalidad de verificar si es posible omitir la segunda punción disminuyendo así el riesgo de complicaciones.

\section{Importancia}

La importancia de este trabajo investigativo radica en la necesidad de disminuir el número de procedimientos invasivos, los cuales representan una causa importante de morbimortalidad materna y fetal.

Adicionalmente, el presente estudio contribuye para establecer los patrones de variación en los valores de beta 2 microglobulina en la orina fetal entre punciones seriadas.

\section{Delimitación}

El estudio se realizó en pacientes embarazadas con edad gestacional mayor o igual de 16 semanas y diagnóstico ecográfico de uropatía obstructiva fetal, que acudieron al servicio de Ginecología y Obstetricia del Centro Médico Docente La Trinidad en el período comprendido entre enero 2005 y octubre 2014.

\section{Marco Teórico}

El desarrollo de las vías genitourinarias se inicia durante la cuarta semana de la gestación con una condensación del mesodermo, la segunda etapa incluye el descenso caudal del mesonefros hasta que entra en contacto con el seno urogenital ${ }^{5}$.

Con el advenimiento de la ecografía obstétrica, empleada en el control normal del embarazo, se han podido detectar precozmente malformaciones fetales de importancia en cerca del $1 \%$ de los embarazos. Las malformaciones del tracto urinario corresponden a un $20 \%$ del total de las malformaciones fetales, ocupando el segundo lugar en importancia después de las del sistema nervioso central, las cuales corresponden al $50 \%$ 6,7.

La gran mayoría de las malformaciones urinarias producen dilatación del tracto urinario, lo que se manifiesta en la ecografía como una hidronefrosis o una ureterohidronefrosis, con o sin megavejiga, pudiendo corresponder a una uropatía obstructiva alta -como estenosis pielouretral, megauréter obstructivo, ureterocele o uréter ectópico-, o a una uropatía obstructiva baja -como valvas uretrales, atresia uretral, síndrome de Prune Belly o vejiga neurogénica ${ }^{7-9}$. Por otra parte, ocurren anomalías cromosómicas en el $23 \%$ de los fetos con uropatía obstructiva ${ }^{3}$. 
En cuanto a la uropatía obstructiva fetal, la lesión obstructiva puede ser uni o bilateral y estar localizada en cualquier punto del tracto urinario. Las anormalidades más comúnmente detectadas son la obstrucción de la unión ureteropélvica, valvas uretrales posteriores, atresia uretral, anomalías de la cloaca y enfermedades renales quísticas.

La historia natural de la uropatía obstructiva depende de la severidad y duración de la obstrucción y el resultado se mide en términos de sobrevida postnatal, hay dos factores importantes que se deben tener en cuenta: la madurez pulmonar y la función renal. Un adecuado volumen de líquido amniótico es necesario para el desarrollo normal del pulmón fetal. La complicación más frecuente del oligohidramnios secundario a obstrucción del tracto urinario es la hipoplasia pulmonar y, en casos severos, la displasia renal final.

Por tanto, cuando se presenta oligohidramnios sin un historial de ruptura de membranas o restricción de crecimiento del feto se debe pensar en una posible anormalidad del tracto urinario. En la uropatía obstructiva fetal, tanto la función renal como el pronóstico, varían de acuerdo con la duración y el grado de obstrucción distal del tracto urinario.

En un feto con sospecha de uropatía obstructiva debe realizarse una confirmación ecográfica para tratar de determinar con más precisión el origen o sitio de la patología, la apariencia del parénquima renal y, por último, la aspiración de orina fetal para cuantificación de los electrolitos y la osmolaridad.

Los avances en el diagnóstico prenatal durante las últimas 2 décadas han mejorado para detectar, caracterizar y tratar anormalidades renales. La ecografía fetal continúa siendo la herramienta de diagnóstico prenatal más importante. La ecografía identifica en el $90 \%$ de los casos a la vejiga desde las 14 semanas de gestación y a los riñones a partir de las 16 semanas de vida intrauterina.

El diagnóstico de malformaciones urinarias es relativamente frecuente, ya que con la ecografía se visualiza fácilmente el contraste entre los tejidos y la orina, en el interior del conducto urinario dilatado, siendo posible con las nuevas resoluciones ecográficas detectar dilataciones hasta de $2 \mathrm{~mm}$ de diámetro. La gran mayoría de las malformaciones urinarias producen dilatación del tracto urinario, lo que se manifiesta en la econografía como una hidronefrosis o una ureterohidronefrosis, con o sin megavejiga, pudiendo corresponder a una uropatía obstructiva alta, baja, o incluso a una condición fisiológica denominada "hidronefrosis transitoria" propia del estado intrauterino. Este último cuadro representa un $20 \%$ a $30 \%$ de las hidronefrosis prenatales y estaría relacionado al ambiente hormonal propio del embarazo, se resuelve en todos los casos durante el primer año de vida, especialmente durante los primeros seis meses.
Mientras la función renal fetal se evalúa a través de la bioquímica urinaria midiendo los electrolitos urinarios, así como los factores proteínicos capaces de identificar la existencia de falla o displasia renal.

La orina fetal se obtiene realizando una vesicocentesis dirigida por ultrasonido. Johnson y col. recomiendan analizar muestras seriadas de orina fetal (al menos 3 ), debido a que los valores de la orina inicial que se encuentra retenida por cierto tiempo no son muy predictivos para determinar la función renal, ni para establecer la tendencia hacia el mejoramiento o el empeoramiento como ayuda para predecir la función renal postnatal debido a la capacidad de la vejiga fetal de concentrar la orina y obtener parámetros que no son representativos de la función renal ${ }^{10}$.

En un esfuerzo para predecir la función renal y para identificar de una manera efectiva la displasia renal, se puede evaluar la bioquímica urinaria. Usualmente la orina fetal es hipotónica; sin embargo, el riñón displásico produce orina isotónica. Es posible predecir con bastante precisión la función renal y el pronóstico usando como parámetros el sodio, cloruro, calcio, la osmolaridad, proteínas totales y los niveles de beta 2 microglobulina de la orina fetal ${ }^{10}$.

Los valores de la biometría urinaria establecidos como parámetros para identificar la función renal normal -ausencia de displasia renal- y un buen pronóstico fetal son los siguientes: sodio menos de $100 \mathrm{mEq} / \mathrm{l}$; cloruro menos de $90 \mathrm{mEq} / \mathrm{l}$; calcio menos de $8 \mathrm{mg} / \mathrm{dl}$; osmolaridad menos de $210 \mathrm{mOsm} /$; beta 2 microglobulina menos de 4 $\mathrm{mg} / \mathrm{dl}$, y proteínas totales menos de $40 \mathrm{mg} / \mathrm{dl}$ ).

La beta 2 microglobulina (beta-2-M) es un polipéptido de bajo peso molecular (11.800 d) que fue identificado por primera vez en la orina de pacientes con enfermedad tubular renal. La beta-2-M es sintetizada por numerosas células y sus concentraciones séricas dependen fundamentalmente de la renovación de la membrana celular y de la velocidad de aclarado, ya que se elimina por filtración glomerular, siendo posteriormente reabsorbida y catabolizada en los túbulos renales proximales ${ }^{11}$.

Los niveles de beta-2-M no cambian con la edad gestacional, su paso transplacentario es improbable, por esta razón sus niveles no están alterados por los maternos, es filtrado por el glomérulo con una reabsorción del 99,9\% por el túbulo renal proximal, por lo que resulta el parámetro más sensible para determinar la función renal fetal, siendo ésta su primera y más conocida aplicación clínica.

La determinación cualitativa de beta-2-M en orina, suero o plasma puede servir como un elemento orientador en el tratamiento de diversas patologías, utilizándose como factor pronóstico en muchas de ellas. La estimación de las concentraciones de beta-2-M en orina fetal ayuda a valorar la función renal del feto en la uropatía obstructiva, permitiendo aconsejar a los padres respecto a la necesidad de intervenir terapéuticamente antes que la condición se agrave ${ }^{12}$. 
La obstrucción urinaria intrauterina severa produce un daño renal progresivo, el cual puede ser evitado mediante una descompresión precoz, lo más pronto posible una vez confirmado el diagnóstico. Afin de evitar este daño permanente han sido desarrollados varios métodos de intervención terapéutica prenatal que buscan aliviar la obstrucción urinaria a través de una corrección quirúrgica primaria o una derivación transitoria, con la idea de posponer el tratamiento específico definitivo. Sin embargo, estos procedimientos tienen riesgos de causar infección, traumatismo fetal, rotura de membrana, corioamnionitis y parto prematuro.

A pesar que la vesicocentesis seriada sería uno de los métodos de descompresión urinaria prenatal, tiene el inconveniente de no mejorar la cantidad de líquido amniótico, y por ser un procedimiento que aumenta la morbilidad materno-fetal, debido al riesgo de infección intrauterina y otras complicaciones propias de la técnica, no se recomienda como tratamiento de la uropatía obstructiva fetal.

Desde el punto de vista terapéutico, la exteriorización de la vejiga y el uréter por medio de cirugía fetal abierta es efectiva, pero las complicaciones son muy frecuentes.

Han sido publicados artículos clínicos informando sobre algunos casos de corrección quirúrgica primaria prenatal, como resecciones de valvas uretrales en fetos por vía percutánea transvesical ${ }^{13}$.

Igualmente, otro método de derivación urinaria fetal usado cada vez con mayor frecuencia cuando hay obstrucción urinaria, son los shunt vesicoamnióticos, consistentes en colocar, por vía percutánea y con guía ecográfica, un catéter interno desde la vejiga fetal a la cavidad amniótica, con la gran ventaja de mejorar el volumen del líquido amniótico y descomprimir la vía urinaria hasta el término del embarazo. Éste es un método permanente que se realiza bajo sedación materno-fetal, no exento de complicaciones, tales como el desplazamiento del catéter, la oclusión del mismo debido a detritus celulares o sobrecrecimiento del tejido de granulación, y la dificultad en su colocación ${ }^{13,14}$.

\section{Hipótesis}

Si se logra determinar un patrón de comportamiento de disminución de beta 2-M en orina fetal, entonces será posible omitir la segunda punción disminuyendo los riesgos asociados a los procedimientos invasivos.

\section{OBJETIVOS}

\section{Objetivo General}

Determinar la variación de los valores de beta 2 microglobulina, en 2 muestras seriadas y consecutivas de orina fetal con intervalos de $48-72$ horas, en fetos con diagnóstico de uropatía obstructiva, de pacientes embarazadas a partir de la semana 16 de gestación que acudieron a la consulta del servicio de Ginecología y Obstetricia del Centro Médico Docente La Trinidad para observar si existe un patrón de disminución con el objetivo de omitir la necesidad de segunda punción.

\section{Objetivos Específicos}

- Cuantificar la diferencia que existe entre 2 muestras seriadas de beta 2 microglobulina en pacientes con uropatía obstructiva fetal.

- Determinar el tratamiento de acuerdo a los valores de funcionalismo renal.

- Verificar la presencia de cromosopatías en muestra de líquido amniótico.

- Demostrar las complicaciones de amniocentesis o vesicocentesis en las pacientes con diagnóstico de uropatía obstructiva fetal.

\section{MÉTODOS}

\section{Tipo de estudio}

Se trata de un estudio clínico unicéntrico, descriptivo y longitudinal.

\section{Población y muestra}

La población estuvo conformada por las pacientes que acudieron al servicio de Ginecología y Obstetricia presentando enfermedad obstructiva renal fetal en el periodo comprendido de enero 2005 a octubre 2014 .

Del tal modo que, la muestra estuvo representada por todas las pacientes que constituyeron la población, luego de haber aplicado los criterios de inclusión y de exclusión.

\section{Criterios de Inclusión}

- Pacientes embarazadas con edad gestacional mayor de 16 semanas.

- Pacientes con fetos que presentan diagnóstico ecográfico de uropatía obstructiva.

- Pacientes con exámenes de laboratorio normales y cultivos (orina, vaginal) negativos.

\section{Criterios de exclusión}

- Pacientes con diagnóstico de cromosopatías.

- Pacientes con punciones intrauterinas previas (amnicentesis o vesicocentesis).

- Pacientes con infecciones activas.

\section{Variables}

De conformidad con los objetivos específicos propuestos para el estudio se consideran las siguientes variables: los valores de beta 2 microglobulina, el tratamiento a seguir, la presencia de cromosopatías, las complicaciones asociadas. 


\section{Procedimiento Clínico}

1.- Evaluación ecográfica del paciente para el diagnóstico de uropatía obstructiva fetal, con determinación de líquido amniótico.

2.- Se le plantea a la paciente la posibilidad de realizar amniocentesis o vesicocentesis para obtención de muestra donde se determinará cariotipo fetal, análisis para sodio, cloruro, osmolaridad, beta 2 microglobulina y proteínas.

3.- Firma del consentimiento informado al haber aceptado la participación en el estudio(Anexo 1).

4.- Realizar amniocentesis o vesicocentesis e indicar reposo absoluto durante 48 horas.

5.- Enviar muestra para estudio de cariotipo fetal y análisis para sodio, cloruro, osmolaridad, beta 2 microglobulina y proteínas.

6.- Análisis de resultados en las primeras 24 - 48 horas, donde de acuerdo a hallazgo de niveles de beta 2 microglobulina, se planteará:

a) Niveles de beta 2 microglobulina mayor a $4 \mathrm{mg} /$ dl realizar segunda vesicocentesis (antes de 72 horas desde la primera vesicocentesis).

b) Niveles de beta 2 microglobulina menor o igual a 4 $\mathrm{mg} / \mathrm{dl}$ colocar derivación vesicoamniótica.

7.- Para pacientes con criterio de segunda vesicocentesis se realizará la misma para determinar sodio, cloruro, osmolaridad, beta 2 microglobulina y proteínas.

8.- Se compara descenso de niveles de beta 2 microglobulina entre la primera y la segunda vesicocentesis.

Los datos obtenidos mediante la evaluación de cada paciente fueron debidamente registrados en un formulario diseñado específicamente para los propósitos del estudio (anexo 2).

\section{Tratamiento estadístico}

Para el análisis estadístico correspondiente se utilizaron pruebas de análisis descriptivo. Con las variables nominales se procedió a calcular sus respectivas frecuencias, tanto absolutas como relativas (porcentajes). Todos los datos obtenidos con el presente estudio se muestran utilizando tablas y fueron graficados con la finalidad de facilitar el análisis estadístico.

\section{RESULTADOS}

El estudio abarcó un total de 15 pacientes embarazadas cuyos fetos tuvieron el diagnóstico de uropatía obstructiva, las cuales cumplieron con los criterios de inclusión y exclusión establecidos, dando su consentimiento informado para participar. A cada paciente se le practicaron 2 vesicocentesis con un intervalo de $48-72$ horas.

Los valores de beta 2 microglobulina resultaron mayores que $4 \mathrm{mg} / \mathrm{dl}$ en todos los 15 casos al realizar la primera vesicocentesis, y disminuyeron a menos de $4 \mathrm{mg} / \mathrm{dl}$ en 7 casos $(46,6 \%)$ en la segunda vesicocentesis, en los cuales se planteó la intervención quirúrgica in utero para colocación de derivaciones vesicoamnióticas (Tabla 1 y Gráfico 1).

La diferencia en los valores de beta 2 microglobulina entre la primera y la segunda vesicocentesis variaron en el rango de 1,57 a 4,04 mg/dl (Tabla 1 y Gráfico 1).

Se observa con atención que, en todos los casos cuando el valor de beta 2 microglobulina resultó menor que $8 \mathrm{mg} / \mathrm{dl}$ en la primera vesicopunción -10 casos en total-, disminuyó invariablemente a valores muy próximos a $4 \mathrm{mg} / \mathrm{dl}$ (máximo de 4,3 $\mathrm{mg} / \mathrm{dl}$ ) en la segunda vesicopunción (Tabla 1 y Gráfico 1).

En los 15 casos evaluados no hubo cromosomopatías. Tampoco ocurrieron complicaciones maternofetales de importancia durante los procedimientos diagnósticos-vesicocentesis- ni durante la colocación del shunt vesicoamniótico en los 5 casos en los que se practicó la terapia descompresiva intrauterina.

Tabla 1. Relación de resultados según valores de beta 2 microglobulina $(\mathrm{mg} / \mathrm{dl})$ comparando primera y segunda vesicocentesis. Centro Médico Docente La Trinidad. Departamento de Ginecología y Obstetricia, entre enero 2005 y octubre 2014.

\begin{tabular}{|c|c|c|c|}
\hline Paciente & $\begin{array}{c}\text { Primera } \\
\text { vesicocentesis }\end{array}$ & $\begin{array}{c}\text { Segunda } \\
\text { vesicocentesis }\end{array}$ & $\begin{array}{c}\begin{array}{c}\text { Diferencia } \\
1^{\mathrm{a}} \quad 2^{\mathrm{a}} \mathrm{v} .\end{array} \\
\end{array}$ \\
\hline 1 & 7,4 & 4,2 & 3,2 \\
\hline 2 & 4,27 & 2,7 & 1,57 \\
\hline 3 & 14,54 & 12,36 & 2,18 \\
\hline 4 & 15,42 & 12,58 & 2,84 \\
\hline 5 & 5,9 & 4,3 & 1,6 \\
\hline 6 & 6,7 & 2,4 & 4,3 \\
\hline 7 & 7,28 & 3,24 & 4,04 \\
\hline 8 & 4,7 & 1,7 & 3,0 \\
\hline 9 & 7,6 & 4,1 & 3,5 \\
\hline 10 & 6,45 & 2,87 & 3,58 \\
\hline 11 & 6,75 & 3,75 & 3,0 \\
\hline 12 & 5,72 & 3,71 & 2,01 \\
\hline 13 & 12,87 & 10,94 & 1,93 \\
\hline 14 & 10,34 & 7,64 & 2,7 \\
\hline 15 & 14,56 & 10,9 & 3,66 \\
\hline
\end{tabular}

Nota: se muestra la diferencia en los valores de beta 2 microglobulina entre la $1^{\mathrm{a}}$ y $2^{\mathrm{a}}$ vesicocentesis, con un rango de variabilidad entre 1,57 y $4,04 \mathrm{mg} / \mathrm{dl}$, y con una media de $2,87 \pm 0,866 \mathrm{mg} / \mathrm{dl}$. 


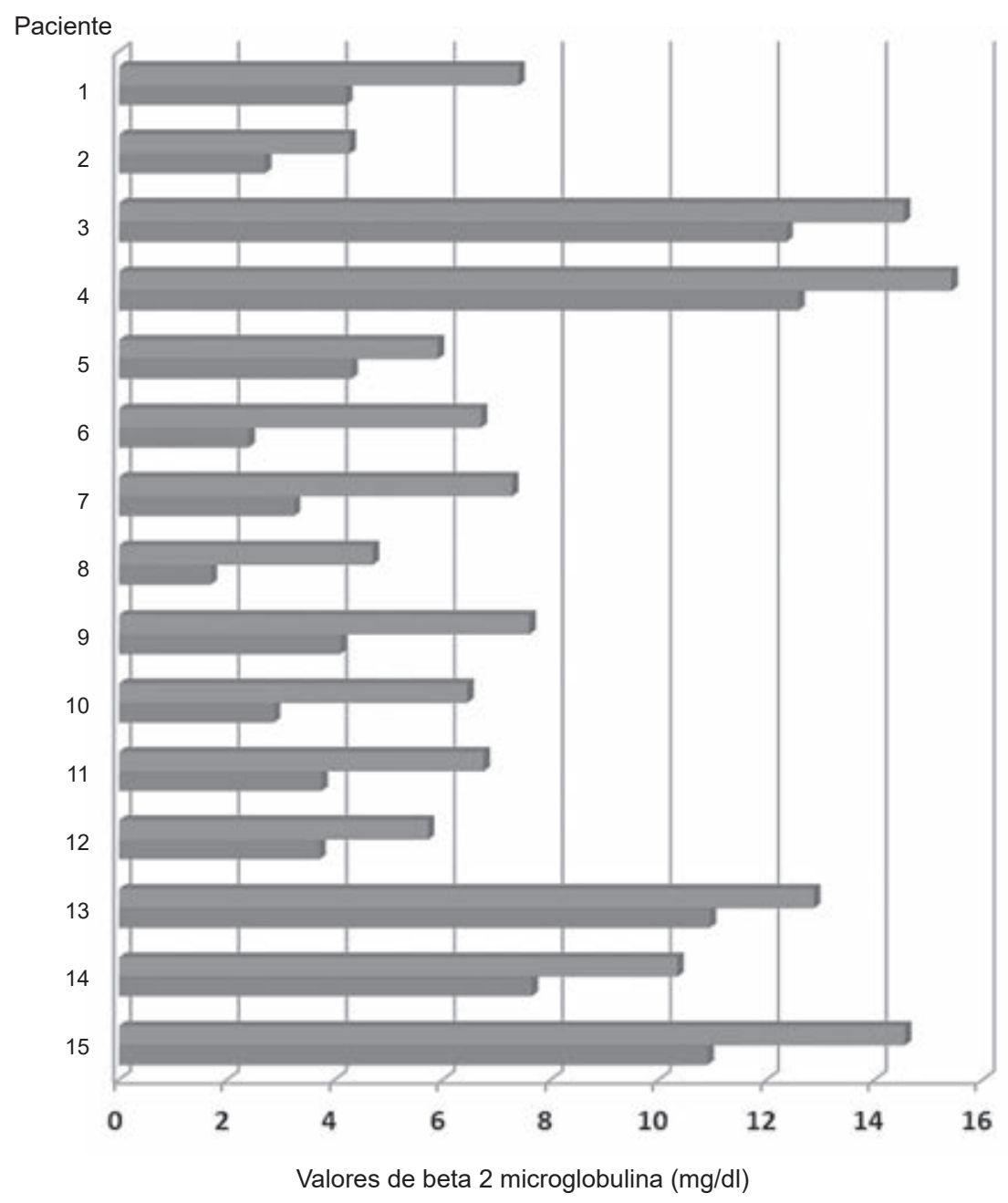

Gráfico 1. Relación de resultados según valores de beta 2 microglobulina $(\mathrm{mg} / \mathrm{dl})$ comparando primera y segunda vesicocentesis. Centro Médico Docente La Trinidad. Departamento de Ginecología y Obstetricia, entre enero 2005 y octubre 2014.

\section{DISCUSIÓN}

Algunas anomalías de los riñones o de las vías excretoras que aparecen in utero pueden comprometer la función renal posteriormente. En el caso de las uropatías obstructivas fetales existen posibilidades terapéuticas precoces. Su aplicación depende de una adecuada evaluación de la función renal y de la determinación del pronóstico evolutivo con base en la búsqueda de signos ecográficos indirectos y en el análisis bioquímico de la orina fetal incluyendo la valoración de marcadores fetales, en especial la determinación de la beta 2 microglobulina ${ }^{15-16}$.

El pronóstico depende del grado de compromiso de la función renal en el momento del diagnóstico. El manejo y tratamiento prenatal de este cuadro, está dirigido a mejorar la función renal y pulmonar a través de la terapia de derivación con la introducción de un catéter entre la vejiga y la cavidad amniótica, previa selección de los candidatos para su intervención in utero basado en el cariotipo fetal, estudio morfológico fetal y evaluación del compromiso renal fetal mediante la obtención y análisis de orina fetal seriadas ${ }^{15}$.

Para fines pronósticos es indispensable determinar la edad gestacional en el momento del diagnóstico, el grado de hidronefrosis, las características del parénquima renal, el compromiso renal bilateral, las alteraciones del líquido amniótico, la función renal fetal y la presencia de otras malformaciones genéticas o congénitas asociadas ${ }^{15}$.

Frente a una hidronefrosis bilateral con oligohidroamnios en el segundo trimestre del embarazo, debe repetirse la ecografía al cabo de una semana, para confirmar la sospecha y comenzar la evaluación de los factores pronósticos, que se van a considerar al tomar una conducta terapéutica. La valoración prenatal se basa en: 1) la ecografía -volumen del líquido amniótico, arquitectura 
del parénquima renal y malformaciones asociadas2) el cariotipo -en orina fetal o líquido amniótico-; y 3) la evaluación de la función renal. Se puede estudiar la función renal fetal por análisis bioquímicos seriados de la orina fetal obtenida por aspiración vesical. Los estudios clínicos han demostrado que la ecografía, la medición de electrolitos urinarios, y muy especialmente los valores de beta 2 microglobulina son buenos predictores de la función renal fetal ${ }^{16,17}$.

La obstrucción urinaria severa durante la etapa intrauterina no sólo produce daño renal progresivo sino también daño pulmonar progresivo. La descompresión temprana, tan pronto se confirma el diagnóstico, permite evitar este daño ya que restablece el volumen del líquido amniótico con la consecuente producción de surfactante, indispensable para la madurez pulmonar ${ }^{18}$.

Se han desarrollado métodos de intervención terapéutica prenatal que persiguen aliviar la obstrucción urinaria por medio de una corrección quirúrgica primaria o una derivación transitoria, con la idea de posponer el tratamiento específico definitivo. No obstante, estos procedimientos de intervención intrauterina implican riesgos de complicaciones materno-fetales ${ }^{18,19}$.

De modo que, la práctica intrauterina de la terapia descompresiva vesicoamniótica -colocación de shuntsólo debe realizarse en casos particulares cuando exista certeza diagnóstica de lesiones renales uni o bilaterales debidas a obstrucción vesical o de las vías urinarias asociadas a oligohidroamnios severos, en feto único, con cariotipo normal, con pruebas de función renal fetal de buen pronóstico y ausencia de otras malformaciones extrarrenales mayores. Cabe señalar que, aún en las condiciones descritas no se puede predecir con certeza el pronóstico renal y pulmonar final, ni las eventuales fallas técnicas y terapéuticas, así como tampoco las complicaciones fetales y maternas asociadas a estos procedimientos ${ }^{18,19}$.

Los padres deben estar bien informados y aceptar los riesgos de estos métodos diagnósticos y terapéuticos. Ellos sólo deben realizarse en instituciones con la capacidad profesional y la experiencia adecuada. Los procedimientos son del manejo de un equipo multidisciplinario conformado por el urólogo pediátrico, obstetra, ecografista, neonatólogo, nefrólogo pediátrico y el ético-médico. Una vez definida la conducta a seguir en cada caso, se debe informar a los padres las ventajas, limitaciones y riesgos de cada procedimiento diagnóstico y terapéutico, respetando su voluntad en la toma de decisiones ${ }^{9,13}$.

En el presente estudio se pudo observar que, en los 10 casos con un valor de beta 2 microglobulina $<8 \mathrm{mg} / \mathrm{dl}$ en la primera vesicocentesis, disminuyó invariablemente a valores muy próximos a $4 \mathrm{mg} / \mathrm{dl}$ (máximo de $4,3 \mathrm{mg} / \mathrm{dl}$ ) en la segunda vesicocentesis. Este hallazgo sugiere la colocación de la derivación vesicoamniótica al obtener valores de beta 2 microglobulina inferiores a $8 \mathrm{mg} / \mathrm{dl}$ en la primera vesicocentesis sin necesidad de realizar una segunda vesicocentesis, reduciéndose de ese modo los riesgos asociados al procedimiento.

\section{CONCLUSIONES}

1. La determinación de beta 2 microglobulina en orina fetal obtenida mediante vesicocentesis seriada fue de gran utilidad clínica en pacientes embarazadas que presentan fetos diagnosticados con uropatía obstructiva para seleccionar tempranamente aquellos casos en los cuales debe adoptarse una conducta terapéutica quirúrgica in utero.

2. En todos los casos cuando beta 2 microglobulina fue $<8 \mathrm{mg} / \mathrm{dl}$ en la primera vesicopunción, disminuyó a valores muy próximos a $4 \mathrm{mg} / \mathrm{dl}$ (máximo de $4,3 \mathrm{mg}$ / dl) en la segunda vesicopunción.

3. Se plantea la colocación in utero de derivaciones vesico - amnióticas en todos los casos de uropatía obstructiva fetal cuando beta 2 microglobulina sea $<8 \mathrm{mg} / \mathrm{dl}$ en la primera vesicopunción, omitiendo realizar una segunda vesicopunción.

4. La terapia de descompresión vesicoamniótica in utero es conveniente en todos los casos de uropatía obstructiva fetal cuando beta 2 microglobulina resulte igual o menor que $4 \mathrm{mg} / \mathrm{dl}$ en la segunda vesicopunción.

\section{RECOMENDACIONES}

- Continuar con la toma de muestras de pacientes para ampliar el valor estadístico del estudio.

- Efectuar el seguimiento neonatal y la evolución de la función renal de cada uno de los pacientes a quienes se les coloque una derivación vesicoamniótica, por parte del departamento de urología pediátrica, a fin de documentar y publicar su comportamiento como base de estudios posteriores.

- Evaluar mediante una investigación posterior más amplia la validez del criterio pronóstico para realizar derivaciones vesicoamnióticas en los casos de uropatía obstructiva fetal cuando el valor de beta 2 microglobulina resulte $<8 \mathrm{mg} / \mathrm{dl}$ en la primera vesicopunción.

Financiamiento: Autofinanciado.

Conflicto de interés: Los autores declaran no tener algún conflicto de interés.

\section{REFERENCIAS BIBLIOGRÁFICAS}

1. McLorie G, Farhat W, Khoury A, Geary D, Ryan G. Outcome analysis of vesicoamniotic shunting in a comprehensive population. J Urol. 2001; 166(3):1036-1040.

2. Crombleholme TM, Harrison MR, Golbus MS, Longaker MT, Langer JC, Callen PW, Anderson RL, Goldstein RB, Filly 
RA. Fetal intervention in obstructive uropathy: prognostic indicators and efficacy of intervention. Am J Obstet Gynecol 1990; 162(5):1239-1244.

3. Skupski DW, Eddleman KA, Zellers N, Ward BE. Rapid exclusion of chromosomal aneuploidies by fluorescence in situ hybridization prior to fetal surgery for obstructive uropathy -a case report. Fetal Diagn Ther. 1994; 9(5):353-356.

4. Lewis KM, Pinckert TL, Cain MP, Ghidini A. Complications of intrauterine placement of a vesicoamniotic shunt. Obstet Gynecol. 1998; 91(5-2):825-827.

5. Valdés A, Pérez H, García R. Embriología humana. 2013; 11:340-348

6. Qureshi F, Jacques SM, Seifman B, Quintero R, Evans MI, Smith C, Johnson MP. In utero fetal urine analysis and renal histology correlate with the outcome in fetal obstructive uropathies. Fetal Diagn Ther. 1996; 11(5):306-312.

7. Baquedano P. Diagnóstico urológico prenatal. Rev Chil Pediatr 2005;76(2);202-206.

8. Berggard I, Bearn AG. Isolation and properties of a low molecular weight 2-globulin occurring in human biological fluids. J Biol Chem 1968; 243:4095-4103.

9. Quispe F, Panozo SV, Verástegui DE, Hochstatter EA, de Guzmán ON, Zegarra W. Síndrome de Prune Belly: diagnóstico y manejo pre y posnatal. Presentación de dos casos. Gac Med Bol 2013; 36(1):35-38.

10. Johnson MP. Bukowski T. In utero surgical treatment of fetal obstructive uropathy: A new comprehensive approach to identify appropriate candidates for vesicoamniotic shunt therapy. Am J Obstet Gynecol. 1994; 170:1770-1779.

11. Bernier GM. 2-microglobulin: Structure, function and significance. VoxSang 1980; 38: 323-327.

12. Lipitz S, Ryan G, Samuell C, Haausler MC, Robson SC, Dhillon HK et al. Fetal urine analysis for the assessment of renal function in obstructive uropathy. Am J Obstet Gynecol 1993; 168:428-435.

13. Evans MI, Sacks AJ, Johnson MP, Robichaux AG, May M, Moghissi KS. Sequential invasive assessment of fetal renal function and the intrauterine treatment of fetal obstructive uropathies. Obstet Gynecol . 1991; 77(4):545-550.

14. Morris RK, Malin GL, Quinlan-Jones E, Middleton LJ, Hemming $\mathrm{K}$, Burke $\mathrm{D}$, et al. Percutaneous vesicoamniotic shunting versus conservative management for fetal lower urinary tract obstruction (PLUTO): a randomised trial. Lancet. 2013; 382(9903):1496-1506.

15. Trnka P, Hiatt MJ, Tarantal AF, Matsell DG. Congenital urinary tract obstruction: defining markers of developmental kidney injury. Ped Res. 2012; 72:446-454.

16. Spaggiari E, Dreux S, Czerkiewicz I, Favre R, Schmitz T, Guimiot $F$ et al. Fetal obstructive uropathy complicated by urinary ascites: outcome and prognostic value of fetal serum $\beta$-2-microglobulin. Ultrasound Obstet Gynecol. 2013; 41(2):185-189.

17. Gazapo E, Gazapo A. Caturla RM. Utilidad clínica de la determinación de beta-2-microglobulina. Medicina Clínica. 1996; 106(19):751-755

18. Hindryckx A, De Catte L. Prenatal diagnosis of congenital renal and urinary tract malformations. Facts Views Vis Obgyn. 2011; 3(3): 165-174.

19. Morris RK, Khan KS, Kilby MD. Vesicoamniotic shunting for fetal lower urinary tract obstruction: an overview. Arch Dis Child Fetal Neonatal Ed 2007; 92(3):166-168.

Correspondencia: Calle Batalla de San Juan 836, dpto. 102, Chacarilla del Estanque, Surco. Lima-Perú.

Correo electrónico:enrique.gil@doctors.org.uk
ORCID iDs

Enrique Gil Guevara

Ramiro Diaz https://orcid.org/0000-0001-6515-2599

https://orcid.org/0000-0001-8458-5488 